\title{
HOLOMORPHIC MOTIONS AND TEICHMÜLLER SPACES
}

\author{
C. J. EARLE, I. KRA, AND S. L. KRUSHKAL' \\ In memory of Halsey L. Royden
}

\begin{abstract}
We prove an equivariant form of Slodkowski's theorem that every holomorphic motion of a subset of the extended complex plane $\widehat{\mathbb{C}}$ extends to a holomorphic motion of $\widehat{\mathbb{C}}$. As a consequence we prove that every holomorphic map of the unit disc into Teichmüller space lifts to a holomorphic map into the space of Beltrami forms. We use this lifting theorem to study the Teichmüller metric.
\end{abstract}

\section{INTRODUCTION}

Let $E$ be a subset of the extended complex plane $\widehat{\mathbb{C}}=\mathbb{C} \cup\{\infty\}$, and let $\Delta$ be the unit disc $\{t \in \mathbb{C} ;|t|<1\}$. By definition, a holomorphic motion of $E$ is a function $f: \Delta \times E \rightarrow \widehat{\mathbb{C}}$ satisfying

(a) $f(0, z)=z$ for all $z \in E$,

(b) $f(\cdot, z)$ is a holomorphic ( $\widehat{\mathbb{C}}$-valued) function on $\Delta$ for each $z \in E$, and

(c) $f(t, \cdot)$ is injective on $E$ for each $t \in \Delta$.

Holomorphic motions were introduced by Mañé, Sad, and Sullivan in [24]. They have been important in the study of dynamical systems ([24], [36], and [38]) and Kleinian groups ([37], [4], and [21]).

Since its inception the study of holomorphic motions has been dominated by the problem of extending a given motion $f: \Delta \times E \rightarrow \widehat{\mathbb{C}}$ to a motion $\tilde{f}: \Delta \times F \rightarrow \widehat{\mathbb{C}}$ of a set $F$ that properly contains $E$. Important partial results were obtained in [24], [39], and [5], and an essentially complete solution was obtained by Slodkowski, who showed in [33] that every $\mathbb{C}$-valued holomorphic motion of a subset of $\mathbb{C}$ can be extended to a $\mathbb{C}$-valued holomorphic motion of $\mathbb{C}$. (It is a routine matter to extend Slodkowski's result to the case of $\widehat{\mathbb{C}}$-valued motions. We indicate one method in the appendix of this paper.) A simpler and more self-contained proof of Slodkowski's theorem (relying on essentially the same basic machinery) has recently been given in [1]. See also [42].

There is an intimate connection between holomorphic motions and Teichmüller spaces, first pointed out by Bers and Royden in [5]. They used results from the theory of Teichmüller spaces to obtain theorems about holomorphic motions. In this paper we reverse that process by using Slodkowski's theorem to obtain new results about Teichmüller spaces. We need an equivariant form of

Received by the editors December 10, 1992 and, in revised form, April 15, 1993.

1991 Mathematics Subject Classification. Primary 32G15; Secondary 30F40.

Research of the first author was supported in part by NSF Grants DMS 8901729 and 9206924; of the second, by NSF Grants DMS 9003361 and 9204092. 
Slodkowski's theorem, which we prove in $\S 2$. (A more general equivariant version of his theorem has been announced independently by Slodkowski in [34].) As an immediate application, we show in $\S 3.1$ that every holomorphic map of the unit disc into Teichmüller space can be lifted to a holomorphic map into the space of Beltrami coefficients. (We remind the reader that in contrast the projection from the space of Beltrami coefficients to Teichmüller space does not admit a holomorphic section [7] unless the image Teichmüller space has dimension zero or one.)

The lifting theorem in $\S 3.1$ seems likely to have numerous applications, some of which we give here. It obviously resolves (in the affirmative) the conjecture in [12] that every holomorphic map of the unit disc into the Teichmüller space $\mathbf{T}(p, 0)$ can be lifted to a holomorphic map into the Teichmüller curve; see $\S 3.2$. It also makes possible a very simple proof, given in $\S 5$, of Royden's theorem (see [30]) on the equality of the Teichmüller and Kobayashi metrics on Teichmüller space (even in the infinite dimensional case). After a brief discussion of extremal Beltrami coefficients, we turn our attention to the study of holomorphic isometries of the unit disc into Teichmüller space. We show that if a holomorphic map from $\Delta$ into Teichmüller space preserves the distance between $\mathrm{t}$ wo distinct points or is an infinitesimal isometry at a single point, then it is a global isometry. The one point case solves a problem posed and partially solved in [31]. Even the two point case is new for infinite dimensional Teichmüller spaces. Finally, we study the uniqueness of geodesics in Teichmüller space and the uniqueness of the lifts of holomorphic maps of the disc into Teichmüller space whose existence is guaranteed by the lifting theorem in $\S 3.1$.

\section{AN EQUIVARIANT VERSION OF SLODKOWSKI'S EXTENSION THEOREM}

The proof of the extension theorem in Slodkowski [33] makes little effort to control the properties of the extended motion, but it can easily be modified to make some control possible. The following theorem is sufficient for our purposes. Slodkowski has independently obtained a different equivariant version of his extension theorem (see [34]).

Theorem 1. Let $E$ be a subset of $\widehat{\mathbb{C}}$ that contains at least three points, and let $G$ be a group of Möbius transformations that map $E$ onto itself. Let $f: \Delta \times E \rightarrow \widehat{\mathbb{C}}$ be a holomorphic motion of $E$. Suppose that for each $g \in G$ and $t \in \Delta$ there is a Möbius transformation $\theta_{t}(g)$ such that

$$
f(t, g(z))=\theta_{t}(g)(f(t, z))
$$

for all $z \in E$. Then $f$ can be extended to a holomorphic motion of $\widehat{\mathbb{C}}$ (still called $f$ ) in such a way that (1) holds for all $g \in G, t \in \Delta$, and $z \in \widehat{\mathbb{C}}$.

Proof. The classical lambda lemma of [24] guarantees that $f$ can be extended to a holomorphic motion of the closure $\bar{E}$ of $E$, and that the extended motion, which we still call $f$, is a continuous map from $\Delta \times \bar{E}$ to $\widehat{\mathbb{C}}$. The set $\bar{E}$ is $G$ invariant, and the continuity of $f$ implies that (1) holds for all $g \in G, t \in \Delta$, and $z \in \bar{E}$. Therefore we shall assume from now on that $E$ is closed.

For each $t \in \Delta$ and $g \in G$, the Möbius transformation $\theta_{t}(g)$ is completely determined by equation (1), since $E$ contains at least three points. It follows easily that for each $t \in \Delta, \theta_{t}$ is a homomorphism of $G$ into the group of 
Möbius transformations and that $\theta_{0}$ is the identity. It also follows that each of the homomorphisms $\theta_{t}$ is injective. Indeed, if $g_{1}$ and $g_{2}$ belong to $G$ and $\theta_{t}\left(g_{1}\right)=\theta_{t}\left(g_{2}\right)$, then equation (1) gives

$$
f\left(t, g_{1}(z)\right)=f\left(t, g_{2}(z)\right) \text { for all } z \in E .
$$

Therefore $g_{1}(z)=g_{2}(z)$ for all $z \in E$, so $g_{1}=g_{2}$.

Identifying the complex Lie group PSL $(2, \mathbb{C})$ with the group of Möbius transformations in the usual way, we obtain for each $g$ in $G$ a map $t \mapsto \theta_{t}(g)$ from $\Delta$ to $\operatorname{PSL}(2, \mathbb{C})$. We claim that each of these maps is holomorphic. To see this, choose three distinct points $z_{1}, z_{2}, z_{3}$ in $E$. For each $t$ in $\Delta$, let $h_{t}$ be the unique Möbius transformation such that

$$
h_{t}\left(z_{j}\right)=f\left(t, z_{j}\right) \text { for } j=1,2,3 .
$$

For any $g$ in $G$, equations (1) and (2) imply

$$
\theta_{t}(g)\left(h_{t}\left(z_{j}\right)\right)=f\left(t, g\left(z_{j}\right)\right) \text { for } j=1,2,3 .
$$

The right-hand sides of equations (2) and (3) are holomorphic functions of $t$ for each $j$, so the maps $t \mapsto h_{t}$ and $t \mapsto \theta_{t}(g) \circ h_{t}$ are holomorphic. Hence so is $t \mapsto \theta_{t}(g)$, as we claimed.

We have proved that the family $\left\{\theta_{t} ; t \in \Delta\right\}$ is a holomorphic family of isomorphisms, in the sense of Bers [4]. Since $\theta_{0}$ is the identity, $\theta_{t}$ is a quasiconformal deformation for all $t$ in $\Delta$, by Proposition 1 of [4]. In other words, for each $t$ in $\Delta$ there is a quasiconformal homeomorphism $f_{t}$ of $\widehat{\mathbb{C}}$ inducing $\theta_{t}$, in the sense that

$$
f_{t} \circ g=\theta_{t}(g) \circ f_{t} \text { for all } g \in G .
$$

In particular each of the isomorphisms $\theta_{t}$ is type preserving.

Let $E^{\prime}$ be any $G$-invariant subset of $\widehat{\mathbb{C}}$, closed or not, that contains $E$, and let $f$ be a holomorphic motion of $E^{\prime}$ extending the given motion of $E$. We will say that $f$ is G-equivariant if (1) holds for all $t$ in $\Delta, g$ in $G$, and $z$ in $E^{\prime}$.

For each $z$ in $\widehat{\mathbb{C}}$ let $G_{z}=\{g \in G ; g(z)=z\}$. Our first goal is to extend $f$ to the $G$-invariant set $E \cup F$, where

$$
F=\left\{z \in \widehat{\mathbb{C}} ; \text { the group } G_{z} \text { is nontrivial }\right\} .
$$

The set $E$ is closed, $G$-invariant, and contains at least three points, so it contains the fixed points of all parabolic and loxodromic (including hyperbolic) transformations $g$ in $G$. (To see this, consider the orbits of points of $E$ under the action of the cyclic group generated by a parabolic or loxodromic $g$ in $G$.) Thus, if $z \in F-E$, the group $G_{z}$ contains only the identity and elliptic transformations. The same is true of each group $\theta_{t}\left(G_{z}\right)$, since the isomorphisms $\theta_{t}$ are type preserving. Therefore all these groups are abelian, and for each $t$ in $\Delta$ the nontrivial elements of $\theta_{t}\left(G_{z}\right)$ all have the same pair of fixed points in $\widehat{\mathbb{C}}$. Since $\theta_{t}(g)$ depends holomorphically on $t$ for each $g$ in $G$, we conclude that for each $z$ in $F-E$ there is a unique holomorphic function $\phi_{z}$ on $\Delta$ such that $\phi_{z}(0)=z$ and $\phi_{z}(t)$ is fixed by $\theta_{t}(g)$ for all $g$ in $G_{z}$ and $t$ in $\Delta$. 
We extend $f$ to $E \cup F$ by putting $f(t, z)=\phi_{z}(t)$ if $t \in \Delta$ and $z \in F-E$. We claim that this extended map $f$ is a $G$-equivariant holomorphic motion of $E \cup F$. We leave it to the reader to check that $f$ is $G$-equivariant and has the defining properties (a) and (b) of a holomorphic motion. The injectivity property (c) is more delicate.

As a first step toward verifying (c) we shall prove

$$
\begin{gathered}
\text { If } f\left(t^{\prime}, z\right)=f\left(t^{\prime}, g(z)\right) \text { for some } g \in G, t^{\prime} \in \Delta, \\
\text { and } z \in E \cup F \text {, then } g \in G_{z} .
\end{gathered}
$$

This is certainly true if $z \in E$, so suppose $z \in F-E$. Put $w=f\left(t^{\prime}, z\right)$ and note that

$$
\theta_{t^{\prime}}(g)(w)=f\left(t^{\prime}, g(z)\right)=w
$$

because of the $G$-equivariance of $f$ and our hypothesis. Choose a quasiconformal homeomorphism $f_{t^{\prime}}$ of $\widehat{\mathbb{C}}$ inducing $\theta_{t}$ and observe that $g$ fixes the point $z^{\prime}=\left(f_{t^{\prime}}\right)^{-1}(w)$. (That is, $g \in G_{z^{\prime}}$.) Similarly, if $h \in G_{z}$, the $G$-equivariance implies that $\theta_{t^{\prime}}(h)$ fixes $w$, so $h \in G_{z^{\prime}}$ and $G_{z} \subset G_{z^{\prime}}$.

We shall assume that $g \notin G_{z}$ (so, in particular, $z^{\prime} \neq z$ ) and obtain a contradiction. Choose a nontrivial $h$ in $G_{z}$. The commutator $h^{*}=h \circ g \circ h^{-1} \circ g^{-1}$ is parabolic, so $z^{\prime}$ is its only fixed point. The transformation $\theta_{t^{\prime}}\left(h^{*}\right)$ is also parabolic and fixes both $f\left(t^{\prime}, z^{\prime}\right)$, by the $G$-equivariance, and $w$, because $f_{t^{\prime}}$ induces $\theta_{t^{\prime}}$. Therefore $f\left(t^{\prime}, z^{\prime}\right)=w\left(=f\left(t^{\prime}, z\right)\right)$. Now, since $h \in G_{z}$ and $G_{z} \subset G_{z^{\prime}}, G$-equivariance implies that $\theta_{t}(h)$ fixes both $f(t, z)$ and $f\left(t, z^{\prime}\right)$ for every $t$ in $\Delta$. But $\theta_{t}(h)$ is always elliptic, and its fixed points are given by two holomorphic functions of $t$ in $\Delta$ with disjoint graphs. Since $f(0, z) \neq f\left(0, z^{\prime}\right)$ we must have $f(t, z) \neq f\left(t, z^{\prime}\right)$ for all $t$ in $\Delta$, contradicting the equality when $t=t^{\prime}$. We have proved (4).

Now we can prove the injectivity property (c). Suppose $f\left(t^{\prime}, z\right)=f\left(t^{\prime}, z^{\prime}\right)$ for some $t^{\prime}$ in $\Delta$ and $z$ and $z^{\prime}$ in $E \cup F$. We must show that $z=z^{\prime}$. That is true if both $z$ and $z^{\prime}$ belong to $E$, so we assume that $z \in F-E$. Now for all $g$ in $G$ we have

$$
f\left(t^{\prime}, g(z)\right)=\theta_{t^{\prime}}(g)\left(f\left(t^{\prime}, z\right)\right)=\theta_{t^{\prime}}(g)\left(f\left(t^{\prime}, z^{\prime}\right)\right)=f\left(t^{\prime}, g\left(z^{\prime}\right)\right),
$$

so $f\left(t^{\prime}, z\right)=f\left(t^{\prime}, g(z)\right)$ if $g \in G_{z^{\prime}}$. By (4) we conclude that $G_{z^{\prime}} \subset G_{z}$, so by symmetry $G_{z^{\prime}}=G_{z}$. Since $z \in F-E, G_{z}$ is a nontrivial abelian group. If $z \neq z^{\prime}$, then $z$ and $z^{\prime}$ are the two fixed points of $G_{z}$, so $f(t, z)$ and $f\left(t, z^{\prime}\right)$ are the two fixed points of $\theta_{t}(g)$ for any $t$ in $\Delta$ and nontrivial $g$ in $G$. This contradicts our assumption that $f\left(t^{\prime}, z\right)=f\left(t^{\prime}, z^{\prime}\right)$, so $z=z^{\prime}$ and (c) is proved. We have extended $f$ to a $G$-equivariant holomorphic motion of $E \cup F$.

Now let $E^{\prime}$ be any $G$-invariant subset of $\widehat{\mathbb{C}}$ such that $E \cup F \subset E^{\prime}$, and let $z_{0} \in \widehat{\mathbb{C}}-E^{\prime}$. Put

$$
E^{\prime \prime}=E^{\prime} \cup\left\{g\left(z_{0}\right) ; g \in G\right\} .
$$

We claim that every $G$-equivariant holomorphic motion of $E^{\prime}$ extends to a $G$-equivariant holomorphic motion of $E^{\prime \prime}$.

To verify this claim, let $f: \Delta \times E^{\prime} \rightarrow \widehat{\mathbb{C}}$ be any $G$-equivariant holomorphic motion of $E^{\prime}$. Extend it (this is possible by the extension theorem of Slodkowski [33]) to a holomorphic motion of $E^{\prime} \cup\left\{z_{0}\right\}$ that we continue to denote 
by $f$. To extend this motion to $E^{\prime \prime}$, we set

$$
f\left(t, g\left(z_{0}\right)\right)=\theta_{t}(g)\left(f\left(t, z_{0}\right)\right) \text { for } t \in \Delta \text { and } g \in G \text {. }
$$

We must show that the extension is a well-defined $G$-equivariant holomorphic motion of the set $E^{\prime \prime}$. If $g_{1}\left(z_{0}\right)=g_{2}\left(z_{0}\right)$ for $g_{1}$ and $g_{2}$ in $G$, then $g_{1}=g_{2}$ (since $\left.z_{0} \notin F\right)$. Thus $f$ is well defined. The $G$-equivariance follows immediately from the definition of $f$, and properties (a) and (b) of a holomorphic motion are again easily verified. In fact, (a) holds by hypothesis for $z \in E^{\prime}$ and by construction for $z=z_{0}$. If $z=g\left(z_{0}\right)$ for some $g$ in $G$, then (a) follows from the fact that $\theta_{0}(g)=g$ for all $g \in G$. Similarly, property (b) is obvious for $z \in E^{\prime} \cup\left\{z_{0}\right\}$ and also holds at $g\left(z_{0}\right)$ for all $g \in G$, since $\theta_{t}(g)$ depends holomorphically on $t$.

Before verifying (c) we make some observations about the fixed points of transformations in $\theta_{t}(G)$, for any given $t$ in $\Delta$. We denote by $F_{g}$ the set of fixed points of any nontrivial $g$ in $G$. For any subset $D$ of $E^{\prime}$, we define

$$
f(t, D)=\{\zeta \in \widehat{\mathbb{C}} ; \zeta=f(t, z) \text { for some } z \in D\} .
$$

For any nontrivial $g$ in $G$, the set $f\left(t, F_{g}\right)$ is contained in the fixed point set of $\theta_{t}(g)$ because $f$ is $G$-equivariant. In fact $f\left(t, F_{g}\right)$ is precisely the fixed point set of $\theta_{t}(g)$ since $\theta_{t}$ is type preserving. Thus, all fixed points of nontrivial elements of $\theta_{t}(G)$ belong to the set $f(t, F)$, which, by hypothesis, is contained in $f\left(t, E^{\prime}\right)$.

To verify (c), assume that $f\left(t, z_{1}\right)=f\left(t, z_{2}\right)$ for some $t$ in $\Delta$ and $z_{1}$ and $z_{2}$ in $E^{\prime \prime}$. We must prove that $z_{1}=z_{2}$. That is true by construction if $z_{1}$ and $z_{2}$ both belong to $E^{\prime} \cup\left\{z_{0}\right\}$, so we assume that $z_{1}=g\left(z_{0}\right)$ for some nontrivial $g$ in $G$. By $G$-equivariance, $f\left(t, z_{0}\right)=f\left(t, g^{-1}\left(z_{2}\right)\right)$. If $z_{2} \in E^{\prime}$, then $f\left(t, g^{-1}\left(z_{2}\right)\right) \in f\left(t, E^{\prime}\right)$. Since $f\left(t, z_{0}\right) \notin f\left(t, E^{\prime}\right)$, we must have $z_{2}=h\left(z_{0}\right)$ for some $h$ in $G$. It follows, by $G$-equivariance, that $f\left(t, z_{0}\right)$ is a fixed point of

$$
\theta_{t}(g)^{-1} \circ \theta_{t}(h)=\theta_{t}\left(g^{-1} \circ h\right) .
$$

Since $f\left(t, z_{0}\right) \notin f\left(t, E^{\prime}\right)$, we conclude that $g=h$ and $z_{1}=z_{2}$, as required. We have proved our claim that $f$ can be extended to a $G$-equivariant holomorphic motion of $E^{\prime \prime}$.

To prove Theorem 1 we simply iterate the construction above, applying it first with $E^{\prime}=E \cup F$, and choosing the successive points $z_{0}$ so that the union of their $G$-orbits is dense in $\widehat{\mathbb{C}}$. Corollary 3 to Theorem 1 in [5] guarantees that the sequence of extended motions converges to the desired $G$-equivariant holomorphic motion of $\widehat{\mathbb{C}}$.

\section{THE LIFTING THEOREMS}

3.1. Lifting to the Beltrami coefficients. Throughout this paper, $\Gamma$ will be a Fuchsian group operating on the upper half plane $\mathbb{H}^{2}$. We shall, as usual, denote by $L^{\infty}\left(\mathbb{H}^{2}, \Gamma\right)$ the complex Banach space of bounded measurable Beltrami differentials for $\Gamma$ supported on $\mathbb{H}^{2}$. Its open unit ball $\mathscr{M}(\Gamma)$ is the space of Beltrami coefficients for $\Gamma$. Let $T(\Gamma)$ be the Teichmüller space of $\Gamma$ and $\Phi$ : $\mathscr{M}(\Gamma) \rightarrow \mathbf{T}(\Gamma)$ the canonical projection. We shall use the Bers embedding (see [14] or [27]) to identify $\mathbf{T}(\Gamma)$ with a subset of the space of bounded holomorphic 
quadratic differentials $\mathbf{B}_{2}(\Gamma)$ for the group $\Gamma$ on the lower half plane $\mathbb{H}_{*}^{2}$. Thus, in particular, $\Phi(\mu) \in \mathbf{B}_{2}(\Gamma)$ for all $\mu$ in $\mathscr{M}(\Gamma)$.

In [7] and [8] it was proved that in general the projection map $\Phi$ has no globally defined holomorphic section. Thus, if we are given a complex manifold $B$ and a holomorphic map $f: B \rightarrow \mathbf{T}(\Gamma)$, there is no guarantee that there is a holomorphic map $g: B \rightarrow \mathscr{M}(\Gamma)$ such that $\Phi \circ g=f$. However, the extension theorem in $\S 2$ leads almost immediately to the following lifting theorem.

Theorem 2. If $f: \Delta \rightarrow \mathbf{T}(\Gamma)$ is a holomorphic map, then there exists a holomorphic $g: \Delta \rightarrow \mathscr{M}(\Gamma)$ such that $\Phi \circ g=f$. If $\mu_{0} \in \mathscr{M}(\Gamma)$ and $\Phi\left(\mu_{0}\right)=f(0)$, we can choose $g$ so that $g(0)=\mu_{0}$.

Proof. Recall that (thanks to the Bers embedding) $f(t) \in \mathbf{B}_{2}(\Gamma)$ for each $t \in \Delta$. Using right translations (and thus perhaps changing the group $\Gamma$ to a quasiconformally conjugate group, see [14] or [27]), we may assume that $f(0)=0$.

Let $\widehat{\mathbb{R}}=\mathbb{R} \cup\{\infty\}$ and $E=\mathbb{H}_{*}^{2} \cup \widehat{\mathbb{R}}$. Observe that $E$ is a $\Gamma$-invariant set. We shall use the map $f$ to define a $\Gamma$-equivariant holomorphic motion of $E$. For each $t \in \Delta$, let $\tilde{f}(t, \cdot)$ be a meromorphic function whose Schwarzian derivative is the quadratic differential $f(t)$. The fact that $f(t) \in \mathbf{T}(\Gamma)$ means that $\tilde{f}(t, \cdot)$ is schlicht on $\mathbb{H}_{*}^{2}$ and has a continuous schlicht extension to $E$. Therefore we can and do specify $\tilde{f}(t, \cdot)$ uniquely by requiring that it fix 0,1 , and $\infty$. Thus, in particular, $\tilde{f}(0, z)=z$ for all $z \in E$.

It is easy to verify that the map

$$
\tilde{f}: \Delta \times E \rightarrow \widehat{\mathbb{C}}
$$

is a holomorphic motion of $E$. In addition, since $f(t)$ is a quadratic differential, for each $t \in \Delta$ and $\gamma \in \Gamma$ there is a Möbius transformation $\theta_{t}(\gamma)$ such that

$$
\tilde{f}(t, \gamma(z))=\theta_{t}(\gamma)(\tilde{f}(t, z))
$$

for all $z \in E$. Thus $\tilde{f}$ is the desired $\Gamma$-equivariant motion.

Using Theorem 1, we extend $\tilde{f}$ to a $\Gamma$-equivariant holomorphic motion $\tilde{f}$ of $\widehat{\mathbb{C}}$. For each $t \in \Delta$, let $g(t)$ be the complex dilatation

$$
g(t)=\tilde{f}_{\bar{z}}(t, \cdot) / \tilde{f}_{z}(t, \cdot)
$$

of $\tilde{f}(t, \cdot)$ in $\mathbb{H}^{2}$. The $\Gamma$-equivariance of $\tilde{f}$ implies that $g(t) \in \mathscr{M}(\Gamma)$ for every $t \in \Delta$, and Theorem 2 of [5] implies that the map $g: \Delta \rightarrow \mathscr{M}(\Gamma)$ is holomorphic. By definition of the Bers embedding, $\Phi(g(t))$ is the Schwarzian derivative of the schlicht function $\tilde{f}(t, \cdot)$ in $\mathbb{H}_{*}^{2}$. Therefore $\Phi \circ g=f$.

The lift $g$ that we have just constructed satisfies $g(0)=0$. If $\mu_{0}$ is a Beltrami coefficient such that $\Phi\left(\mu_{0}\right)=0$, let $h: \mathscr{M}(\Gamma) \rightarrow \mathscr{M}(\Gamma)$ be the right translation that carries 0 to $\mu_{0}$. Then $\Phi \circ h=\Phi$, so $h \circ g$ is a lift of $f$ that maps 0 to $\mu_{0}$.

Remark 1. Suppose the holomorphic map $f: \Delta \rightarrow \mathbf{T}(\Gamma)$ with $f(0)=0$ is an isometry with respect to the Poincaré metric on $\Delta$ and the Teichmüller metric on $\mathbf{T}(\Gamma)$. (These metrics are defined in $\S \S 4$ and 5.) If the dimension of $\mathbf{T}(\Gamma)$ is finite, it is not hard to prove that $f$ has a unique holomorphic lift $g: \Delta \rightarrow \mathscr{M}(\Gamma)$ satisfying $g(0)=0$. We shall prove a more general uniqueness theorem in $\S 8$. 
Remark 2. According to a remark of Bers and Royden [5] (see the final sentence and Proposition 4 in that paper), Slodkowski's extension theorem in [33] implies Theorem 2 in the special case that the group $\Gamma$ has type $(0, n)$, so that $\mathbf{T}(\Gamma)$ is the Teichmüller space $\mathbf{T}(0, n)$ of the $n$-times punctured sphere. The general case of Theorem 2 depends on our equivariant version (Theorem 1) of Slodkowski's theorem.

3.2. Lifting to the Teichmüller curve. In the work of the first two authors on choosing points on varying Riemann surfaces of finite analytic type [12] the following problem was proposed. Let $f$ be a holomorphic map from a complex manifold $B$ into the Teichmüller space $\mathbf{T}(p, 0)$. (Here $\mathbf{T}(p, n)$ is, of course, the Teichmüller space of Riemann surfaces of finite analytic type $(p, n)$.) Determine all holomorphic maps $h: B \rightarrow \mathbf{V}(p, 0)$ such that $\pi \circ h=f$. (Here $\pi: \mathbf{V}(p, n) \rightarrow \mathbf{T}(p, n)$ is the universal Teichmüller curve of Riemann surfaces of finite analytic type $(p, n) ; \mathbf{V}(p, n)^{\prime} \subset \mathbf{V}(p, n)$ is the total space of the corresponding punctured curve. The fibers of $\mathbf{V}(p, n)$ over $\mathbf{T}(p, n)$ are compact, while each fiber of $\mathbf{V}(p, n)^{\prime}$ has $n$ punctures. In particular $\mathbf{V}(p, 0)=\mathbf{V}(p, 0)^{\prime}$.) In [12] it was conjectured that for $B=\Delta$, a lift $h$ always exists. The lifting theorem of the previous subsection immediately implies that conjecture.

Theorem 3. If $f: \Delta \rightarrow \mathbf{T}(p, n)$ is a holomorphic map, then there exists a holomorphic $h: \Delta \rightarrow \mathbf{V}(p, n)^{\prime}$ such that $\pi \circ h=f$.

Proof. Choose a torsion-free Fuchsian group $\Gamma$ of type $(p, n)$, so that $\mathbf{T}(p, n)$ $=\mathbf{T}(\Gamma)$. The canonical projection $\Phi$ from $\mathscr{M}(\Gamma)$ to $\mathbf{T}(p, n)$ can be written in the form $\Phi=\pi \circ \Psi$, where $\Psi: \mathscr{M}(\Gamma) \rightarrow \mathbf{V}(p, n)^{\prime}$ is holomorphic and $\pi$ : $\mathbf{V}(p, n)^{\prime} \rightarrow \mathbf{T}(p, n)$ is the restriction of the projection map of the Teichmüller curve to the punctured Teichmüller curve (see [19] or [27]). By Theorem 2, there is a holomorphic map $g: \Delta \rightarrow \mathscr{M}(\Gamma)$ such that $\Phi \circ g=f$. The required map $h=\Psi \circ g$.

Remark 3. It is easy to see that every map $h$ satisfying the requirements of Theorem 3 has the above form $h=\Psi \circ g$. In fact the universal covering space of $\mathbf{V}(p, n)^{\prime}$ is naturally isomorphic to $\mathbf{T}(p, n+1)$, and we can write $\Psi=\varpi \circ \phi$, where $\phi: \mathscr{M}(\Gamma) \rightarrow \mathbf{T}(p, n+1)$ is a canonical projection and $\varpi: \mathbf{T}(p, n+1) \rightarrow$ $\mathbf{V}(p, n)^{\prime}$ is a holomorphic universal covering (see [19] or [27]). Given $h$ as in Theorem 3, choose a lift $\tilde{h}: \Delta \rightarrow \mathbf{T}(p, n+1)$. By Theorem 2 there is a holomorphic map $g: \Delta \rightarrow \mathscr{M}(\Gamma)$ such that $\tilde{h}=\phi \circ g$. Clearly $\Psi \circ g=h$.

Thus the description of the lifts $h: \Delta \rightarrow \mathbf{V}(p, n)^{\prime}$ of a given $f$ is completely reduced to a description of the lifts $g: \Delta \rightarrow \mathscr{M}(\Gamma)$ of the same map. We shall say more about the problem of describing the lifts $g$ in $\S \S 8$ and 9.

\section{Metrics IN $\mathscr{M}(\Gamma)$}

4.1. Teichmüller's metric. As in [14] we define the Teichmüller metric $\tau_{\mathscr{M}(\Gamma)}$ on $\mathscr{M}(\Gamma)$ by

$$
\tau_{\mathscr{M}(\Gamma)}(\mu, \nu)=\tanh ^{-1}\left\|\frac{\mu-\nu}{1-\bar{\mu} \nu}\right\|_{\infty} .
$$

for $\mu$ and $\nu$ in $\mathscr{M}(\Gamma)$. 
The right side of (5) equals

$$
\text { ess } \sup \left\{d_{\Delta}(\mu(z), \nu(z)) ; z \in \mathbb{H}^{2}\right\},
$$

where $d_{\Delta}$ is the Poincaré metric (of curvature -4 ) on $\Delta$, defined by

$$
d_{\Delta}(z, \zeta)=\tanh ^{-1}\left|\frac{z-\zeta}{1-\bar{z} \zeta}\right|
$$

for $z$ and $\zeta$ in $\Delta$. It is therefore not surprising that the metric $\tau_{\mathscr{M}(\Gamma)}$ is induced by the Finsler structure $F_{\mathscr{M}(\Gamma)}$ on $\mathscr{M}(\Gamma)$ defined by

$$
F_{\mathscr{M}(\Gamma)}(\mu, \nu)=\left\|\frac{\nu}{1-|\mu|^{2}}\right\|_{\infty},
$$

for $\mu$ in $\mathscr{M}(\Gamma)$ and $\nu$ in $L^{\infty}\left(\mathbb{H}^{2}, \Gamma\right)$. The easy proof that this Finsler structure induces the Teichmüller metric on $\mathscr{M}(\Gamma)$ is given in [11]. (The reader should note that the Teichmüller metric in [11] differs by a factor of two from the metric defined here.)

4.2. Kobayashi's metric. Let $X$ be any connected complex manifold modelled on a complex Banach space, and let $H(\Delta, X)$ be the set of holomorphic maps of $\Delta$ into $X$. The Kobayashi function $\delta_{X}: X \times X \rightarrow[0,+\infty]$ is defined for all $x$ and $y$ in $X$ by

$$
\delta_{X}(x, y)=\inf \left\{d_{\Delta}(0, t) ; f(0)=x \text { and } f(t)=y \text { for some } f \in H(\Delta, X)\right\},
$$

provided the set of maps described above is nonempty and $+\infty$ otherwise. It is obvious from this definition that if $X$ and $Y$ are connected complex Banach manifolds and $f: X \rightarrow Y$ is a holomorphic map, then

$$
\delta_{Y}\left(f\left(x_{1}\right), f\left(x_{2}\right)\right) \leq \delta_{X}\left(x_{1}, x_{2}\right) \text { for all } x_{1}, x_{2} \in X,
$$

with equality if $f$ is biholomorphic.

By definition, the Kobayashi (pseudo)metric $d_{X}$ on $X$ is the largest (pseudo)metric on $X$ such that

$$
d_{X}(x, y) \leq \delta_{X}(x, y) \text { for all } x, y \in X .
$$

In particular, if $\delta_{X}$ itself is a metric, as will be the case in our examples, then $d_{X}$ and $\delta_{X}$ are equal.

The following proposition implies that the Teichmüller and Kobayashi metrics on $\mathscr{M}(\Gamma)$ coincide. It is essentially a special case of a general result of L.A. Harris (see Exercise 6 on p. 394 of [16]), but we shall provide the proof for the reader's convenience.

Proposition 1 (Harris). For all $\mu$ and $\nu$ in $\mathscr{M}(\Gamma)$ we have

$$
\delta_{\mathscr{M}(\Gamma)}(\mu, \nu)=\tau_{\mathscr{M}(\Gamma)}(\mu, \nu) .
$$

Proof. We may assume that $\mu \neq \nu$. Assume first that $\mu=0$. Suppose that $f \in H(\Delta, \mathscr{M}(\Gamma)), f(0)=0$, and $f(t)=\nu$ for some $t$ in $\Delta$. Then, by the 
Schwarz lemma (see p. 100 of [18] or p. 184 of [17]),

$$
|t| \geq\|f(t)\|_{\infty}=\|\nu\|_{\infty} .
$$

Taking the infimum over all such $f$ we obtain

$$
\delta_{\mathscr{M}(\Gamma)}(0, \nu) \geq d_{\Delta}\left(0,\|\nu\|_{\infty}\right)=\tau_{\mathscr{M}(\Gamma)}(0, \nu) .
$$

To obtain the opposite inequality we choose the particular function $f(t)=$ $t \nu /\|\nu\|_{\infty}$ and observe that

$$
\delta_{\mathscr{M}(\Gamma)}(0, \nu) \leq d_{\Delta}\left(0,\|\nu\|_{\infty}\right)=\tau_{\mathscr{M}(\Gamma)}(0, \nu),
$$

since $f\left(\|\nu\|_{\infty}\right)=\nu$. That proves the proposition if $\mu=0$.

If $\mu \neq 0$, observe that the function $f: \mathscr{M}(\Gamma) \rightarrow L^{\infty}\left(\mathbb{H}^{2}, \Gamma\right)$ defined by

$$
f(\lambda)=\frac{\mu-\lambda}{1-\bar{\mu} \lambda}
$$

for all $\lambda$ in $\mathscr{M}(\Gamma)$, is a biholomorphic map of $\mathscr{M}(\Gamma)$ onto itself. Therefore

$$
\delta_{\mathscr{M}(\Gamma)}(\mu, \nu)=\delta_{\mathscr{M}(\Gamma)}(f(\mu), f(\nu))=\tau_{\mathscr{M}(\Gamma)}(0, f(\nu)),
$$

which equals $\tau_{\mathscr{M}(\Gamma)}(\mu, \nu)$, by $(5)$.

\section{Metrics IN $T(\Gamma)$ AND THE ROYDEN-GARDINER THEOREM}

5.1. Teich:müller's metric. The Teichmüller metric (5) and Finsler structure (7) on $\mathscr{M}(\Gamma)$ induce a quotient metric and Finsler structure on $\mathbf{T}(\Gamma)$. To be more explicit, the Teichmüller metric $\tau_{\mathbf{T}(\Gamma)}$ is given by

$$
\begin{array}{r}
\tau_{\mathbf{T}(\Gamma)}(\varphi, \psi)=\inf \left\{\tau_{\mathscr{M}(\Gamma)}(\mu, \nu) ; \mu \text { and } \nu \text { in } \mathscr{M}(\Gamma),\right. \\
\Phi(\mu)=\varphi, \text { and } \Phi(\nu)=\psi\},
\end{array}
$$

for all $\varphi$ and $\psi$ in $\mathbf{T}(\Gamma)$, and the infinitesimal Teichmüller metric $F_{\mathbf{T}(\Gamma)}$ is given by

$$
F_{\mathbf{T}(\Gamma)}(\Phi(\mu), \psi)=\inf \left\{F_{\mathscr{M}(\Gamma)}(\mu, \nu) ; \nu \in L^{\infty}\left(\mathbb{H}^{2}, \Gamma\right) \text { and } \Phi^{\prime}(\mu) \nu=\psi\right\},
$$

for all $\mu \in \mathscr{M}(\Gamma)$ and $\psi \in \mathbf{B}_{2}(\Gamma)$ ). (Recall that we are using the Bers embedding to interpret the canonical projection $\Phi: \mathscr{M}(\Gamma) \rightarrow \mathbf{T}(\Gamma)$ as a holomorphic map onto an open subset $\mathbf{T}(\Gamma)$ of $\mathbf{B}_{2}(\Gamma)$.)

According to a general theorem of O'Byrne [28], the distance function induced on $\mathbf{T}(\Gamma)$ by the Finsler structure (11) is the Teichmüller metric (10); see also $§ 7.2$ of [14].

5.2. The Royden-Gardiner theorem. An important theorem of Royden [30] states that on the Teichmüller spaces $\mathbf{T}(p, 0)$ the Teichmüller metric and Kobayashi function are equal (and hence the Teichmüller and Kobayashi metrics coincide). Royden's theorem was ultimately extended to all Teichmüller spaces by Gardiner (see Chapter 7 of [14]). Our first lifting theorem provides an almost trivial proof of this important result. 
Theorem 4 (Royden-Gardiner). For all Fuchsian groups $\Gamma$ and all $\varphi$ and $\psi$ in $\mathrm{T}(\Gamma)$, we have

$$
\tau_{\mathbf{T}(\Gamma)}(\varphi, \psi)=\delta_{\mathbf{T}(\Gamma)}(\varphi, \psi) .
$$

In particular, the Teichmüller and Kobayashi metrics of $\mathrm{T}(\Gamma)$ coincide.

Proof. Fix any $\varphi$ and $\psi$ in $\mathbf{T}(\Gamma)$. The inequality (9) implies immediately that $\delta_{\mathbf{T}(\Gamma)}(\varphi, \psi) \leq \inf \left\{\delta_{\mathscr{M}(\Gamma)}(\mu, \nu) ; \mu\right.$ and $\nu$ in $\mathscr{M}(\Gamma), \Phi(\mu)=\varphi$, and $\left.\Phi(\nu)=\psi\right\}$.

Hence, by Proposition 1 and formula (10),

$$
\delta_{\mathbf{T}(\Gamma)}(\varphi, \psi) \leq \tau_{\mathbf{T}(\Gamma)}(\varphi, \psi) .
$$

For the opposite inequality, choose $f$ in $H(\Delta, \mathbf{T}(\Gamma))$ so that $f(0)=\varphi$ and $f(t)=\psi$ for some $t$ in $\Delta$. Use Theorem 2 to write $f=\Phi \circ g$ with $g$ in $H(\Delta, \mathscr{M}(\Gamma))$. Again using Proposition 1, we have

$$
d_{\Delta}(0, t) \geq \tau_{\mathscr{M}(\Gamma)}(g(0), g(t)) \geq \tau_{\mathbf{T}(\Gamma)}(\Phi(g(0)), \Phi(g(t)))=\tau_{\mathbf{T}(\Gamma)}(\varphi, \psi) .
$$

Taking the infimum over all such $f$ and using (8) we get

$$
\delta_{\mathbf{T}(\Gamma)}(\varphi, \psi) \geq \tau_{\mathbf{T}(\Gamma)}(\varphi, \psi) .
$$

\section{Extremal Beltrami differentials}

6.1. The Hamilton-Krushkal' condition. We call the Beltrami coefficient $\mu \in$ $\mathscr{M}(\Gamma)$ extremal if

$$
\tau_{\mathscr{M}(\Gamma)}(0, \mu)=\tau_{\mathbf{T}(\Gamma)}(0, \Phi(\mu))
$$

or (equivalently)

$$
\|\mu\|_{\infty}=\inf \left\{\|\nu\|_{\infty} ; \nu \in \mathscr{M}(\Gamma) \text { and } \Phi(\nu)=\Phi(\mu)\right\} .
$$

We call the Beltrami differential $\mu \in L^{\infty}\left(\mathbb{H}^{2}, \Gamma\right)$ infinitesimally extremal if

$$
F_{\mathscr{M}(\Gamma)}(0, \mu)=F_{\mathbf{T}(\Gamma)}\left(0, \Phi^{\prime}(0) \mu\right)
$$

or (equivalently)

$$
\|\mu\|_{\infty}=\inf \left\{\|\nu\|_{\infty} ; \nu \in L^{\infty}\left(\mathbb{H}^{2}, \Gamma\right) \text { and } \Phi^{\prime}(0) \nu=\Phi^{\prime}(0) \mu\right\}
$$

Let $\mathbf{Q}(\Gamma)$ be the Banach space of integrable holomorphic quadratic differentials for $\Gamma$ (defined on $\mathbb{H}^{2}$ ), and let $\mathbf{Q}(\Gamma)^{*}$ be its dual space. Let $P$ : $L^{\infty}\left(\mathbb{H}^{2}, \Gamma\right) \rightarrow \mathbf{Q}(\Gamma)^{*}$ be the linear transformation of norm one defined by putting

$$
(P \mu)(\varphi)=\iint_{\mathbb{H}^{2} / \Gamma} \mu(z) \varphi(z)\left|\frac{d z \overline{d z}}{2}\right| \quad \text { if } \mu \in L^{\infty}\left(\mathbb{H}^{2}, \Gamma\right) \text { and } \varphi \in \mathbf{Q}(\Gamma)
$$

It is well known (see for instance pp. 155-157 of [10]) that $P \mu=0$ if and only if $\Phi^{\prime}(0) \mu=0$. That fact and the Hahn-Banach theorem give

$$
\|P \mu\|=\inf \left\{\|\nu\|_{\infty} ; P \nu=P \mu\right\}=\inf \left\{\|\nu\|_{\infty} ; \Phi^{\prime}(0) \nu=\Phi^{\prime}(0) \mu\right\} .
$$

Comparison with (14) yields the following useful fact (see [28]). 
Proposition 2. A Beltrami differential $\mu \in L^{\infty}\left(\mathbb{H}^{2}, \Gamma\right)$ is infinitesimally extremal if and only if it satisfies the Hamilton-Krushkal' condition $\|P \mu\|=\|\mu\|_{\infty}$.

This condition owes its name to the following important proposition, which is the end result of deep and important work by at least four mathematicians. Hamilton [15] and Krushkal' [23] established necessity of this condition for extremality (in this connection see also [3] and [28]). Sufficiency when the group $\Gamma$ is trivial was established by Reich and Strebel [29], and Strebel [35] showed how the arguments in [29] extend to the general case. A good reference for the proposition in full generality is Chapter 6 of Gardiner's book [14].

Proposition 3. A Beltrami coefficient $\mu \in \mathscr{M}(\Gamma)$ is extremal if and only if it satisfies the Hamilton-Krushkal' condition.

6.2. An application of Schwarz's lemma. The following lemma will be crucial to our study of isometric holomorphic maps from $\Delta$ (with its Poincaré metric) to $T(\Gamma)$ (with the Teichmüller metric). It also provides a flexible method for obtaining new extremal Beltrami coefficients from a given one (see the proof of Theorem 6 in $\S 8$ ).

Lemma 1. Let $g: \Delta \rightarrow \mathscr{M}(\Gamma)$ be a holomorphic map with $g(0)=0$. The following conditions are equivalent:

(a) there is $t^{\prime}$ in $\Delta-\{0\}$ such that $g\left(t^{\prime}\right)$ is extremal and $\left\|g\left(t^{\prime}\right)\right\|_{\infty}=\left|t^{\prime}\right|$,

(b) $g^{\prime}(0)$ is infinitesimally extremal and $\left\|g^{\prime}(0)\right\|_{\infty}=1$, and

(c) for all $t$ in $\Delta, g(t)$ is extremal and $\|g(t)\|_{\infty}=|t|$.

Proof. Let $P: L^{\infty}\left(\mathbb{H}^{2}, \Gamma\right) \rightarrow \mathbf{Q}(\Gamma)^{*}$ be the linear map (15) and let $h=P \circ g$. Then $\|h(t)\| \leq\|g(t)\|_{\infty}<1$ for all $t$ in $\Delta$, and $h(0)$ and $g(0)$ are both 0 . We can therefore apply Schwarz's lemma to both $g$ and $h$.

Now assume (a). By (a) and Proposition 3, $\left\|h\left(t^{\prime}\right)\right\|=\left\|g\left(t^{\prime}\right)\right\|_{\infty}=\left|t^{\prime}\right|$ for some $t^{\prime}$ in $\Delta-\{0\}$. This is the case of equality in Schwarz's lemma, so (see p. 184 of [17])

$$
\left\|h^{\prime}(0)\right\|=\left\|g^{\prime}(0)\right\|_{\infty}=1 \text {. }
$$

Since $h^{\prime}(0)=P\left(g^{\prime}(0)\right)$, Proposition 2 says that (17) is equivalent to condition (b). Thus (a) implies (b).

Now assume (b). This means (17) holds. Again this is the case of equality in Schwarz's lemma, so for all $t$ in $\Delta$ we have

$$
\|h(t)\|=\|g(t)\|_{\infty}=|t| .
$$

That proves (c), since $h(t)=P(g(t))$.

Finally, (c) trivially implies (a).

\section{HOLOMORPHIC ISOMETRIES FROM $\Delta$ INTO $\mathbf{T}(\Gamma)$}

Recall that $H(\Delta, \mathbf{T}(\Gamma))$ is the set of holomorphic maps of $\Delta$ into $\mathbf{T}(\Gamma)$. For any $f$ in $H(\Delta, \mathbf{T}(\Gamma))$ the Royden-Gardiner theorem gives

$$
\tau_{\mathbf{T}(\Gamma)}\left(f(t), f\left(t^{\prime}\right)\right) \leq d_{\Delta}\left(t, t^{\prime}\right)
$$

for all $t$ and $t^{\prime}$ in $\Delta$. Letting $t^{\prime}$ approach $t$ in (18) we get the inequality

$$
F_{\mathbf{T}(\Gamma)}\left(f(t), f^{\prime}(t)\right) \leq \frac{1}{1-|t|^{2}},
$$


for all $t$ in $\Delta$ (and $f$ as above). We call $f$ in $H(\Delta, \mathbf{T}(\Gamma))$ a holomorphic isometry if equality holds in (18), and hence also in (19), for all $t$ and $t^{\prime}$ in $\Delta$.

If $\mathbf{T}(\Gamma)$ is finite dimensional, $f \in H(\Delta, \mathbf{T}(\Gamma))$, and equality holds in (18) at a single pair of distinct points of $\Delta$, then Teichmüller's theorem implies easily that $f$ is a holomorphic isometry. The question whether equality in (19) at a single point also implies that $f$ is a holomorphic isometry (still in the finite dimensional case) was studied by Royden in [31], and he obtained some positive results in that direction. Our lifting theorem allows us to give an affirmative answer in all cases, even if the dimension of $\mathbf{T}(\Gamma)$ is infinite.

Theorem 5. Let $f: \Delta \rightarrow \mathbf{T}(\Gamma)$ be holomorphic and let $t_{1} \in \Delta$. Suppose either that

$$
\tau_{\mathbf{T}(\Gamma)}\left(f\left(t_{1}\right), f\left(t_{2}\right)\right)=d_{\Delta}\left(t_{1}, t_{2}\right) \text { for some } t_{2} \neq t_{1}
$$

or

$$
F_{\mathbf{T}(\Gamma)}\left(f\left(t_{1}\right), f^{\prime}\left(t_{1}\right)\right)=\frac{1}{1-\left|t_{1}\right|^{2}}
$$

Then $f$ is a holomorphic isometry.

Proof. We shall prove first that the two point equality (20) implies the infinitesimal equality (21). Since we can precede $f$ by a holomorphic isometry of $\Delta$ and follow $f$ by a right translation (an isometric and holomorphic map) from $\mathbf{T}(\Gamma)$ to $\mathbf{T}\left(\Gamma^{\prime}\right)$ (for some group $\Gamma^{\prime}$ quasiconformally conjugate to $\Gamma$ ), we may assume that $t_{1}=0$ in $\Delta$ and that $f(0)=0$ in $\mathbf{T}(\Gamma)$. By Theorem 2, we can choose a holomorphic map $g: \Delta \rightarrow \mathscr{M}(\Gamma)$ so that $g(0)=0$ and $\Phi \circ g=f$. Assume (20). Then

$$
d_{\Delta}\left(0, t_{2}\right)=\tau_{\mathbf{T}(\Gamma)}\left(0, f\left(t_{2}\right)\right) \leq \tau_{\mathscr{M}(\Gamma)}\left(0, g\left(t_{2}\right)\right) \leq d_{\Delta}\left(0, t_{2}\right),
$$

so

$$
\tau_{\mathbf{T}(\Gamma)}\left(0, f\left(t_{2}\right)\right)=\tau_{\mathscr{M}(\Gamma)}\left(0, g\left(t_{2}\right)\right)=d_{\Delta}\left(0, t_{2}\right) .
$$

The first equality says that $g\left(t_{2}\right)$ is extremal; the second implies that $\left\|g\left(t_{2}\right)\right\|_{\infty}=$ $\left|t_{2}\right|$, by equations (5) and (6). Thus $g$ satisfies condition (a) of Lemma 1 , so condition (b) also holds. Condition (b) says precisely that

$$
1=\left\|g^{\prime}(0)\right\|_{\infty}=\left\|P\left(g^{\prime}(0)\right)\right\| \text {. }
$$

Now (16) and the chain rule give

$$
\left\|P\left(g^{\prime}(0)\right)\right\|=\inf \left\{\|\nu\|_{\infty} ; \Phi^{\prime}(0) \nu=\Phi^{\prime}(0)\left(g^{\prime}(0)\right)\right\}=\inf \left\{\|\nu\|_{\infty} ; \Phi^{\prime}(0) \nu=f^{\prime}(0)\right\} .
$$

Therefore (using (11)) we can rewrite condition (b) as

$$
1=\left\|g^{\prime}(0)\right\|_{\infty}=F_{\mathbf{T}(\Gamma)}\left(0, f^{\prime}(0)\right),
$$

which implies (21) since $t_{1}=0$ and $f(0)=0$.

Next we shall prove that the infinitesimal equality (21) implies not only (20) but the much stronger equation

$$
\tau_{\mathbf{T}(\Gamma)}\left(f\left(t_{1}\right), f(t)\right)=d_{\Delta}\left(t_{1}, t\right) \text { for all } t \in \Delta .
$$


Again we may assume without loss of generality that $t_{1}=0$ in $\Delta$ and $f(0)=0$ in $\mathbf{T}(\Gamma)$. Choose $g: \Delta \rightarrow \mathscr{M}(\Gamma)$ as above and observe that

$$
F_{\mathbf{T}(\Gamma)}\left(0, f^{\prime}(0)\right) \leq\left\|g^{\prime}(0)\right\|_{\infty} \leq 1,
$$

by (11) and Schwarz's lemma. Now $F_{\mathbf{T}(\Gamma)}\left(0, f^{\prime}(0)\right)=1$, by $(21)$, so $g$ satisfies equation (22). We saw above that (22) is condition (b) of Lemma 1 , so $g$ satisfies the equivalent condition (c). In other words

$$
\tau_{\mathbf{T}(\Gamma)}(0, f(t))=\tau_{\mathbf{T}(\Gamma)}(0, \Phi(g(t)))=\tau_{\mathscr{M}(\Gamma)}(0, g(t))=d_{\Delta}(0, t)
$$

for all $t$ in $\Delta$. Since $t_{1}=0$ and $f(0)=0$, this implies (23).

We have proved that for any $t_{1}$ in $\Delta$ equation (23) follows from either (20) or $(21)$. Now choose any $t^{\prime}$ in $\Delta$. To complete the proof we must verify the equation

$$
\tau_{\mathbf{T}(\Gamma)}\left(f\left(t^{\prime}\right), f(t)\right)=d_{\Delta}\left(t^{\prime}, t\right) \text { for all } t \text { in } \Delta .
$$

If $t^{\prime}=t_{1}$ there is nothing to prove, so we assume $t^{\prime} \neq t_{1}$. In that case the known equation

$$
\tau_{\mathbf{T}(\Gamma)}\left(f\left(t^{\prime}\right), f\left(t_{1}\right)\right)=d_{\Delta}\left(t^{\prime}, t_{1}\right) .
$$

is a two point equality with $t^{\prime}$ in the position formerly occupied by $t_{1}$. As we have already seen, that two point equality implies the required equation (24).

The finite dimensional case of the theorem solves the problem posed by Royden [31]. We state this result formally as

Corollary 1. Assume that $\mathbf{T}(\Gamma)$ is finite dimensional. Let $f: \Delta \rightarrow \mathbf{T}(\Gamma)$ be holomorphic and let $t_{1} \in \Delta$. Suppose that either (20) or (21) holds. Then $f$ is a holomorphic isometry, and its image is a Teichmüller disc.

Proof. The only statement that we have not proved is that in the finite dimensional case the image of a holomorphic isometry is a Teichmüller disc. This is a well-known consequence of Teichmüller's theorem. We shall not offer a proof here since we shall prove a substantially more general result in the next section.

Our second corollary answers a question raised in [20]. Let $\Gamma$ be a finitely generated torsion free Fuchsian group (of the first kind) of type $(p, n)$. Let $\pi: \mathscr{F}(\Gamma) \rightarrow \mathbf{T}(\Gamma)$ be the Bers fiber space over the Teichmüller space $\mathbf{T}(\Gamma)$. The base space of this fibration is $\mathbf{T}(p, n)$; the total space is isomorphic to $\mathbf{T}(p, n+1)$, and therefore carries a Teichmüller metric. Assume now that $(p, n) \neq(0,3)$. It was shown in [20] (also in [26]) that the fibers $\pi^{-1}(x), x \in$ $\mathbf{T}(\Gamma)$ are not Teichmüller discs in $\mathscr{F}(\Gamma)$ (they are, of course, conformal discs). In fact it was shown that for all $x \in \mathbf{T}(\Gamma)$

$$
\tau_{\mathscr{F}(\Gamma)}\left(t_{1}, t_{2}\right)<d_{\Delta}\left(t_{1}, t_{2}\right) \text { for all } t_{1} \text { and } t_{2} \in \pi^{-1}(x) \text { with } t_{1} \neq t_{2} \text {. }
$$

However, the question of whether there is a similar strict inequality between the infinitesimal metrics remained open. Now we can prove

Corollary 2. Let $\Gamma$ be a torsion free Fuchsian group of type $(p, n) \neq(0,3)$. For every $x \in \mathbf{T}(\Gamma)$, the restriction to $\pi^{-1}(x)$ of the infinitesimal Teichmüller metric on $\mathscr{F}(\Gamma)$ is strictly less than the infinitesimal Poincaré metric on this disc. 
Proof. As usual it is no loss of generality to assume that $x=0$ in $\mathbf{T}(\Gamma)$, so that $\pi^{-1}(x)=\pi^{-1}(0)$. If the infinitesimal metrics were equal at some point, Theorem 5 would imply that the map $f(t)=(0, t)$ from $\mathbb{H}^{2}$ to $\mathscr{F}(\Gamma)$ is a holomorphic isometry onto its image $\pi^{-1}(0)$, contradicting (25).

\section{UNIQUENESS OF GEODESICS}

By definition, a geodesic segment $J$ in $\mathbf{T}(\Gamma)$ is the image of an injective continuous map $f$ from a nontrivial compact real interval $[a, b]$ into $\mathbf{T}(\Gamma)$ such that

$$
\tau_{\mathbf{T}(\Gamma)}\left(f\left(x_{1}\right), f\left(x_{3}\right)\right)=\tau_{\mathbf{T}(\Gamma)}\left(f\left(x_{1}\right), f\left(x_{2}\right)\right)+\tau_{\mathbf{T}(\Gamma)}\left(f\left(x_{2}\right), f\left(x_{3}\right)\right)
$$

whenever $a \leq x_{1} \leq x_{2} \leq x_{3} \leq b$. The points $f(a)$ and $f(b)$ are called the endpoints of $J$ and are determined (as an unordered pair) by $J$. We say that the geodesic segment $J$ joins the points $\varphi_{1}$ and $\varphi_{2}$ in $\mathbf{T}(\Gamma)$ if they are the endpoints of $J$. Any closed connected subset of a geodesic segment is a geodesic segment (provided that it contains more than one point).

Given two distinct points $\varphi_{1}$ and $\varphi_{2}$ in $T(\Gamma)$ there is always at least one geodesic segment joining them. To see this we may (as usual) assume that $\varphi_{1}=0$ and we can choose an extremal Beltrami coefficient $\mu \neq 0$ in $\mathscr{M}(\Gamma)$ with $\Phi(\mu)=\varphi_{2}$. The image of the map $f:[0,\|\mu\|] \rightarrow \mathbf{T}(\Gamma)$ defined by

$$
f(t)=\Phi\left(t \mu /\|\mu\|_{\infty}\right),
$$

for $t$ in $[0,\|\mu\|]$ is a geodesic segment joining the given points. In fact, if we extend $f$ to $\Delta$ by using formula (26) for all $t$ in $\Delta$, the resulting function (as can be seen, for instance, from Theorem 5) is a holomorphic isometry from $\Delta$ to $\mathbf{T}(\Gamma)$.

We call $\mu \in \mathscr{M}(\Gamma)$ a uniquely extremal Beltrami coefficient if

$$
\Phi(\nu) \neq \Phi(\mu) \text { whenever } \nu \in \mathscr{M}(\Gamma), \quad \nu \neq \mu, \text { and }\|\nu\|_{\infty} \leq\|\mu\|_{\infty} .
$$

In particular, every uniquely extremal $\mu$ is extremal.

When $\mathbf{T}(\Gamma)$ is finite dimensional every extremal $\mu$ is uniquely extremal and has the special form

$$
\mu=k|\varphi| / \varphi,
$$

where $0 \leq k<1$ and $\varphi \in \mathbf{Q}(\Gamma)-\{0\}$. (See Chapter 6 of [14].) Moreover, every geodesic segment is uniquely determined by its endpoints, and every holomorphic isometry $f: \Delta \rightarrow \mathbf{T}(\Gamma)$ with $f(0)=0$ has the form (26), with $\mu$ given by (27). The image of such a holomorphic isometry is called a Teichmüller disc.

When $\mathbf{T}(\Gamma)$ is infinite dimensional, an extremal $\mu$ is not necessarily uniquely extremal, and there always exist two points that are the endpoints of infinitely many distinct geodesic segments. This was proved by Li Zhong (see [41]) when the group $\Gamma$ is trivial and by Tanigawa (see [40]) in the general case. However, Li Zhong proved (see Theorem 3 in [41]) that the geodesic segment joining 0 and $\Phi(\mu)$ is unique if $\mu$ is uniquely extremal and $|\mu|$ is constant. The following theorem includes the converse of Li Zhong's result. It also implies the statements in the preceding paragraph about uniqueness of geodesic segments and holomorphic isometries in the finite dimensional case. 
Theorem 6. Suppose $\mu$ in $\mathscr{M}(\Gamma)$ is extremal and nonzero. The following four conditions are equivalent:

(a) $\mu$ is uniquely extremal and $|\mu|=\|\mu\|_{\infty}$ a.e.,

(b) there is only one geodesic segment joining 0 and $\Phi(\mu)$,

(c) there is only one holomorphic isometry $f: \Delta \rightarrow \mathbf{T}(\Gamma)$ such that $f(0)=0$ and $f\left(\|\mu\|_{\infty}\right)=\Phi(\mu)$, and

(d) there is only one holomorphic map $g: \Delta \rightarrow \mathscr{M}(\Gamma)$ such that $g(0)=0$ and $\Phi\left(g\left(\|\mu\|_{\infty}\right)\right)=\Phi(\mu)$.

Proof. Li Zhong proved in [41] that (a) implies (b). (Li Zhong assumes that the group $\Gamma$ is trivial, but his proof works equally well in the general case.) To prove that (b) implies (c), suppose $f_{1}$ and $f_{2}$ are holomorphic isometries both of which carry 0 in $\Delta$ to 0 in $T(\Gamma)$ and $\|\mu\|_{\infty}$ in $\Delta$ to $\Phi(\mu)$ in $\mathbf{T}(\Gamma)$. Condition (b) implies that $f_{1}=f_{2}$ on the line segment $\left[0,\|\mu\|_{\infty}\right]$, hence everywhere in $\Delta$. Thus, (b) implies (c).

Next we prove that (a) implies (d). Suppose $g: \Delta \rightarrow \mathscr{M}(\Gamma)$ is a holomorphic map such that $g(0)=0$ and $\Phi\left(g\left(\|\mu\|_{\infty}\right)\right)=\Phi(\mu)$. By Schwarz's lemma,

$$
\left\|g\left(\|\mu\|_{\infty}\right)\right\|_{\infty} \leq\|\mu\|_{\infty}
$$

The unique extremality of $\mu$ therefore implies that $g\left(\|\mu\|_{\infty}\right)=\mu$. Now $\lambda=$ $\mu /\|\mu\|_{\infty}$ is an extreme point of the closed unit ball in $L^{\infty}\left(\mathbb{H}^{2}, \Gamma\right)$ since, by $(a)$, $|\lambda|=1$ almost everywhere. We can therefore use the strong form of Schwarz's lemma (see p. 95 of [6]) to conclude that

$$
g(t)=t \lambda \text { for all } t \in \Delta .
$$

The proof (as in [6]) goes as follows. Let $h(0)=g^{\prime}(0)$ and $h(t)=g(t) / t$ for $t$ in $\Delta-\{0\}$. Then $h$ is a holomorphic map from $\Delta$ into the closed unit ball of $L^{\infty}\left(\mathbb{H}^{2}, \Gamma\right)$. Since $\lambda$ is an extreme point of that ball and $h\left(\|\mu\|_{\infty}\right)=\lambda$, the strong maximum modulus theorem (Proposition 6.19 of [6]) says that $h(t)=\lambda$ for all $t$ in $\Delta$ and $g$ has the required form. Since this uniquely determines $g$, (a) implies (d).

Observe that if the dimension of $\mathbf{T}(\Gamma)$ is finite, Teichmüller's theorem implies that (a) holds, and hence the remaining three conditions hold as well. Thus, in the finite dimensional case, Theorem 6 is an easy (and familiar) consequence of Teichmüller's theorem. For the remainder of the proof we shall assume that $\mathbf{T}(\Gamma)$ is infinite dimensional.

We prove next that (d) implies (c). Let $f: \Delta \rightarrow \mathbf{T}(\Gamma)$ be a holomorphic isometry that sends 0 and $\|\mu\|_{\infty}$ to the required points. Using Theorem 2, choose a holomorphic map $g: \Delta \rightarrow \mathscr{M}(\Gamma)$ such that $g(0)=0$ and $\Phi \circ g=f$. Then $\Phi\left(g\left(\|\mu\|_{\infty}\right)\right)=\Phi(\mu)$, so the uniqueness condition (d) implies that

$$
g(t)=t \mu /\|\mu\|_{\infty} \text { for all } t \in \Delta .
$$

Therefore $f(t)$ is given by equation (26) for all $t$ in $\Delta$, and (d) implies (c).

It remains to prove that (c) implies (a). We shall prove first that if (c) holds, then $|\mu|=\|\mu\|_{\infty}$ almost everywhere. Fix $r$ in the open interval $(0,1)$ and let $E_{r}$ be the ( $\Gamma$-invariant) set $\left\{z \in \mathbb{H}^{2} ;|\mu(z)|<r\|\mu\|_{\infty}\right\}$. We need to prove that $E_{r}$ has measure zero. Choose a nontrivial $\varphi$ in $\mathbf{Q}(\Gamma)$. Let $\chi_{r}$ be the 
characteristic function of $E_{r}$. For $t$ in $\Delta$, put

$$
f_{1}(t)=\Phi\left(t \mu /\|\mu\|_{\infty}\right)
$$

and

$$
f_{r}(t)=\Phi\left(\left(t \mu /\|\mu\|_{\infty}\right)+\frac{1-r}{2} t\left(t-\|\mu\|_{\infty}\right)\left(\chi_{r}|\varphi| / \varphi\right)\right) .
$$

These functions are holomorphic maps from $\Delta$ to $\mathbf{T}(\Gamma)$ sending 0 to 0 and $\|\mu\|_{\infty}$ to $\Phi(\mu)$, so they are holomorphic isometries, by Theorem 5. Hence, by (c), they coincide. Therefore

$$
0=f_{1}^{\prime}(0)-f_{r}^{\prime}(0)=\frac{1-r}{2}\|\mu\|_{\infty} \Phi^{\prime}(0)\left(\chi_{r}|\varphi| / \varphi\right) .
$$

Therefore $P\left(\chi_{r}|\varphi| / \varphi\right)=0$ so, by (15),

$$
P\left(\chi_{r}|\varphi| / \varphi\right)(\varphi)=\iint_{E_{r} / \Gamma}|\varphi(z)|\left|\frac{d z \overline{d z}}{2}\right|=0 .
$$

Since the zeros of $\varphi$ are isolated, $E_{r}$ must have measure zero, as required.

Finally we must show that (c) implies that $\mu$ is uniquely extremal. The proof is inspired by Li Zhong's computations on pp. 271-272 of [41]. We must introduce more notation. If $f$ is a quasiconformal homeomorphism of $\mathbb{H}^{2}$ onto itself, we define its complex dilatation $\mu_{f}$ by the formula

$$
\mu_{f}=f_{\bar{z}} / f_{z} .
$$

If $f$ and $g$ are two such homeomorphisms, the chain rule implies the useful formula

$$
\mu_{f \circ g}=\frac{\mu_{g}+\left(\mu_{f} \circ g\right) \alpha_{g}}{1+\bar{\mu}_{g}\left(\mu_{f} \circ g\right) \alpha_{g}}
$$

with $\alpha_{g}=\left(\left|g_{z}\right| / g_{z}\right)^{2}$

If $\nu$ is any Beltrami coefficient, we denote by $w_{\nu}$ the unique quasiconformal homeomorphism of $\mathbb{H}^{2}$ onto itself whose complex dilatation is $\nu$ and whose continuous extension to the closure of $\mathbb{H}^{2}$ (still denoted by $w_{\nu}$ ) fixes the points 0,1 , and $\infty$.

Now assume (c). Suppose $\|\nu\|_{\infty} \leq\|\mu\|_{\infty}$ and $\Phi(\nu)=\Phi(\mu)$. We must show that $\nu=\mu$. Since $\mu$ is extremal, so is $\nu$, and $\|\nu\|_{\infty}=\|\mu\|_{\infty}$. By Theorem 5, $f(t)=\Phi\left(t \nu /\|\nu\|_{\infty}\right)$ is a holomorphic isometry, and by (c)

$$
\Phi\left(t \nu /\|\nu\|_{\infty}\right)=\Phi\left(t \mu /\|\mu\|_{\infty}\right) \text { for all } t \in \Delta .
$$

This observation has two useful consequences. First, we use (c) with the extremal Beltrami coefficient $\nu$ in the role of $\mu$, concluding (as above) that

$$
|\nu|=\|\nu\|_{\infty}\left(=\|\mu\|_{\infty}\right) \text { almost everywhere. }
$$

Second, we observe that $\Phi(s \nu)=\Phi(s \mu)$ if $0<s<1$. Equivalently, $w_{s \nu}=w_{s \mu}$ on $\mathbb{R}$ for all $s$ in $(0,1)$. Therefore, for any fixed $s$ in $(0,1)$ we have

$$
w_{\mu} \circ\left(w_{s \mu}\right)^{-1} \circ w_{s \nu}=w_{\mu} \text { on } \mathbb{R} \text {. }
$$

The left side of (30) defines a quasiconformal homeomorphism $w_{\lambda}$ of $\mathbb{H}^{2}$ onto itself, and (30) says that $\Phi(\lambda)=\Phi(\mu)$. To compute $\lambda$ we shall use 
equation (28) twice. To simplify the resulting formulas we write $\|\mu\|_{\infty}=k$ and $s k=k^{\prime}$. Recall that $|\mu|=k$ almost everywhere. First we use (28) with $f=w_{\mu} \circ\left(w_{s \mu}\right)^{-1}$ and $g=w_{s \mu}$, so that $f \circ g=w_{\mu}$. Solving for $\mu_{f}$ and taking absolute values we find that

$$
\left|\mu_{f}\right|=\left(k-k^{\prime}\right) /\left(1-k k^{\prime}\right)
$$

almost everywhere. Put $k^{\prime \prime}=\left(k-k^{\prime}\right) /\left(1-k k^{\prime}\right)$ and observe that

$$
k=\left(k^{\prime}+k^{\prime \prime}\right) /\left(1+k^{\prime} k^{\prime \prime}\right) \text {. }
$$

We next use (28) with $f$ as before but $g=w_{s \nu}$, so that $f \circ g=w_{\lambda}$. Thus $\lambda$ is given by the right-hand side of (28). Taking absolute values and using equations (29), (31), and (32) we find that

$$
|\lambda| \leq \frac{\left|\mu_{g}\right|+\left|\mu_{f} \circ g\right|}{1+\left|\mu_{g}\right|\left|\mu_{f} \circ g\right|}=\frac{k^{\prime}+k^{\prime \prime}}{1+k^{\prime} k^{\prime \prime}}=k\left(=\|\mu\|_{\infty}\right)
$$

almost everywhere. Since $\Phi(\lambda)=\Phi(\mu)$ and $\mu$ is extremal, $\lambda$ is also extremal. Therefore (c) holds with $\lambda$ in the role of $\mu$, and we conclude that $|\lambda|=k$ almost everywhere. This can happen only if $\left(\mu_{f} \circ g\right) \alpha_{g}$, and hence $\lambda$, is a positive multiple of $\mu_{g}(=s \nu)$ almost everywhere. We conclude that $\lambda=\nu$ almost everywhere, so

$$
w_{\nu}=w_{\lambda}=w_{\mu} \circ\left(w_{s \mu}\right)^{-1} \circ w_{s \nu}
$$

and

$$
w_{\nu} \circ\left(w_{s \nu}\right)^{-1}=w_{\mu} \circ\left(w_{s \mu}\right)^{-1}
$$

everywhere in $\mathbb{H}^{2}$. Letting $s$ approach 0 we find that $w_{\nu}=w_{\mu}$ everywhere in $\mathbb{H}^{2}$, so $\nu=\mu$ as required.

Remark 4. When the dimension of $\mathbf{T}(\Gamma)$ is finite, the implications (a) $\Rightarrow(\mathrm{b})$ and $(\mathrm{b}) \Rightarrow$ (c) tell us that every geodesic segment is uniqueiy determined by its endpoints and that the image of any holomorphic isometry is a Teichmüller disc.

Remark 5. In [41] Li Zhong asks whether condition (b) above holds whenever $\mu$ is a uniquely extremal Beltrami coefficient. In view of Theorem 6, Li Zhong's question is equivalent to the question whether every uniquely extremal $\mu$ has the property that $|\mu|=\|\mu\|_{\infty}$ almost everywhere. This is well known to be a difficult problem.

Remark 6. The functions $f_{r}$ in the proof that (c) implies (a) can be used to show that there are infinitely many distinct geodesic segments with endpoints $\Phi(0)$ and $\Phi(\mu)$ whenever $\mu$ is extremal and the set where $|\mu|$ is less than $\|\mu\|$ has positive measure. A construction of such Beltrami coefficients $\mu$ is given by Tanigawa in [40].

\section{UNIQUENESS OF LIFTINGS}

If the extremal Beltrami coefficient $\mu$ satisfies condition (a) of Theorem 6, then condition (d) is also satisfied, so the holomorphic isometry $f: \Delta \rightarrow \mathbf{T}(\Gamma)$ defined by equation (26) has a unique (holomorphic) lifting $g: \Delta \rightarrow \mathscr{M}(\Gamma)$ with $g(0)=0$. One might hope that this is the only case in which such liftings 
are unique, but the following theorem provides numerous counterexamples to such a conjecture. (See Corollary 3.)

Let $\mathrm{T}(1)$ and $\mathscr{M}(1)$ be the Teichmüller space and space of Beltrami coefficients of the trivial Fuchsian group (which consists of the identity alone), respectively. Thus, if $\Gamma$ is any Fuchsian group, $\mathbf{T}(\Gamma)$ is a subset of $\mathbf{T}(1)$, $\mathscr{M}(\Gamma)$ is a subset of $\mathscr{M}(1)$, and $\Phi: \mathscr{M}(\Gamma) \rightarrow \mathrm{T}(\Gamma)$ is the restriction to $\mathscr{M}(\Gamma)$ of $\Phi: \mathscr{M}(1) \rightarrow \mathbf{T}(1)$. The space $\mathbf{T}(1)$ is the universal Teichmüller space of Bers.

Theorem 7. Suppose the Fuchsian group $\Gamma$ contains no elliptic transformations. Let $f: \Delta \rightarrow \mathbf{T}(\Gamma)$ be a holomorphic map. Suppose there is exactly one holomorphic map $g: \Delta \rightarrow \mathscr{M}(\Gamma)$ such that $g(0)=0$ and $\Phi \circ g=f$. Then $g$ is the only holomorphic map from $\Delta$ into $\mathscr{M}(1)$ such that $g(0)=0$ and $\Phi \circ g=f$.

Proof. Suppose $h: \Delta \rightarrow \mathscr{M}(1)$ is a holomorphic map such that $h(0)=0$, $\Phi \circ h=f$, but $h \neq g$. We shall obtain a contradiction. Let $\tilde{h}$ and $\tilde{g}$ be the holomorphic motions of $\widehat{\mathbb{C}}$ defined respectively by

$$
\tilde{h}(t, z)=w^{h(t)}(z)
$$

and

$$
\tilde{g}(t, z)=w^{g(t)}(z)
$$

(As usual, $w^{\mu}$ is the quasiconformal homeomorphism of $\widehat{\mathbb{C}}$ that fixes the points 0,1 , and $\infty$ and has complex dilatation $\mu$ in $\mathbb{H}^{2}$ and 0 in $\mathbb{H}_{*}^{2}$.)

Choose $t_{0}$ in $\Delta$ so that $h\left(t_{0}\right) \neq g\left(t_{0}\right)$, and choose $z_{0}$ in $\widehat{\mathbb{C}}$ so that

$$
\tilde{h}\left(t_{0}, z_{0}\right) \neq \tilde{g}\left(t_{0}, z_{0}\right) \text {. }
$$

Since $\Phi \circ h=\Phi \circ g$, we have

$$
\tilde{h}(t, z)=\tilde{g}(t, z) \text { for all }(t, z) \in \Delta \times \mathbb{H}_{*}^{2} .
$$

Therefore $z_{0} \in \mathbb{H}^{2}$.

Since $g$ maps $\Delta$ into $\mathscr{M}(\Gamma)$, for each $t \in \Delta$ and $\gamma \in \Gamma$ there is a Möbius transformation $\theta_{t}(\gamma)$ such that

$$
\tilde{g}(t, \gamma(z))=\theta_{t}(\gamma)(\tilde{g}(t, z))
$$

for all $z \in \widehat{\mathbb{C}}$ (and in particular for $z \in \mathbb{H}_{*}^{2}$ ). As in the proof of Theorem 1, we define a holomorphic motion $\tilde{g}^{*}$ of the $\Gamma$-invariant set

$$
E^{\prime \prime}=\mathbb{H}_{*}^{2} \cup\left\{\gamma\left(z_{0}\right) ; \gamma \in \Gamma\right\}
$$

by setting $\tilde{g}^{*}=\tilde{g}$ in $\Delta \times \mathbb{H}_{*}^{2}$ and

$$
\tilde{g}^{*}\left(t, \gamma\left(z_{0}\right)\right)=\theta_{t}(\gamma)\left(\tilde{h}\left(z_{0}\right)\right) .
$$

By Theorem 1 , we can extend $\tilde{g}^{*}$ to a holomorphic motion of $\widehat{\mathbb{C}}$ so that

$$
\tilde{g}^{*}(t, \gamma(z))=\theta_{t}(\gamma)\left(\tilde{g}^{*}(t, z)\right)
$$

for all $t \in \Delta, \gamma \in \Gamma$, and $z \in \widehat{\mathbb{C}}$. 
As in the proof of Theorem 2, we obtain a holomorphic map $g^{*}: \Delta \rightarrow \mathscr{M}(\Gamma)$ by putting

$$
g^{*}(t)=\tilde{g}_{\bar{z}}^{*}(t, \cdot) / \tilde{g}_{z}^{*}(t, \cdot) .
$$

Since

$$
w^{g^{*}(t)}(z)=\tilde{g}^{*}(t, z)=\tilde{g}(t, z)=w^{g(t)}(z)
$$

for all $z \in \mathbb{H}_{*}^{2}$, we have $\Phi \circ g^{*}=\Phi \circ g$. Therefore, by our hypothesis on $g, g^{*}=g$. This in turn implies that $\tilde{g}^{*}=\tilde{g}$ everywhere in $\Delta \times \widehat{\mathbb{C}}$. But, by construction,

$$
\tilde{g}^{*}\left(t_{0}, z_{0}\right)=\tilde{h}\left(t_{0}, z_{0}\right) \neq \tilde{g}\left(t_{0}, z_{0}\right) .
$$

That is the required contradiction.

Corollary 3. There is a holomorphic map $f: \Delta \rightarrow \mathbf{T}(1)$ such that there is exactly one holomorphic map $g: \Delta \rightarrow \mathscr{M}(1)$ satisfying $g(0)=0$ and $\Phi \circ g=f$, but $f$ is not a holomorphic isometry.

Proof. Let $\Gamma$ be the group of cover transformations of a holomorphic universal covering map $\pi: \mathbb{H}^{2} \rightarrow X$, where $X$ is a Riemann surface of finite type. Let $f: \Delta \rightarrow \mathbf{T}(\Gamma)$ be a holomorphic isometry with $f(0)=0$. Then $f$ satisfies the hypothesis (and conclusion) of the last theorem. But $f$ is not a holomorphic isometry when viewed as a map into $\mathbf{T}(1)$, since the inclusion map from $\mathbf{T}(\Gamma)$ to $\mathbf{T}(1)$ contracts Teichmüller distances. (See McMullen [25].)

Remark 7. The first author has constructed a rather complicated example of a holomorphic map $f: \Delta \rightarrow \mathbf{T}(1)$ with the properties stated in the above corollary and with the additional property that it cannot be interpreted as a holomorphic isometry into a smaller Teichmüller space. It is still of the form $f(t)=\Phi\left(t \mu /\|\mu\|_{\infty}\right)$, where $\mu \in \mathscr{M}(1)$ has the property that $|\mu|=\|\mu\|_{\infty}$ almost everywhere. Every known example of an $f$ satisfying the conditions of the corollary has that special form.

\section{NONEXISTENCE OF LIFTINGS}

Theorem 2 depends very much on the fact that the domain of the holomorphic map $f$ is $\Delta$. If the domain of $f$ is two-dimensional, the lifting theorem may fail, as the following example shows.

Theorem 8. Let $\Gamma$ be a Fuchsian group with signature $(1,2 ; n, n), 2 \leq n<$ $\infty$, and let $A, B, C, D$ be canonical generators, so that $\Gamma$ has the presentation

$$
A \circ B \circ A^{-1} \circ B^{-1} \circ C \circ D=C^{n}=D^{n}=I \text {. }
$$

Let $\alpha: \Gamma \rightarrow \mathbb{Z} / n \mathbb{Z}$ be the homomorphism that satisfies

$$
\alpha(A)=\alpha(B)=0, \alpha(C)=1 \text {, and } \alpha(D)=-1 .
$$

The kernel of $\alpha$ is a closed surface group $\Gamma_{n}$ of genus $n$, and the inclusion map of $\mathbf{T}(\Gamma)$ in $\mathbf{T}\left(\Gamma_{n}\right)$ cannot be lifted to a holomorphic map of $\mathbf{T}(\Gamma)$ into $\mathscr{M}\left(\Gamma_{n}\right)$.

Proof. First we shall verify that $\Gamma_{n}$ is a closed surface group of genus $n$. Since every elliptic transformation in $\Gamma$ is conjugate to a power of $C$ or $D$, the kernel $\Gamma_{n}$ of $\alpha$ contains no elliptic transformations. Therefore the quotient map from $\mathbb{H}^{2}$ to the Riemann surface $\mathbb{H}^{2} / \Gamma_{n}$ is a holomorphic universal covering. The natural projection from $\mathbb{H}^{2} / \Gamma_{n}$ to $\mathbb{H}^{2} / \Gamma$ is a branched covering map with 
a compact image. Since this map has finite degree $n, \mathbb{H}^{2} / \Gamma_{n}$ is a compact Riemann surface. To compute its genus we observe that

$$
\operatorname{Area}\left(\mathbb{H}^{2} / \Gamma_{n}\right)=n \operatorname{Area}\left(\mathbb{H}^{2} / \Gamma\right)=2 \pi(2 n-2) \text {. }
$$

Here we have used the formula on p. 233 of [13] to express the area of $\mathbb{H}^{2} / \Gamma$ (with respect to the area measure induced by the hyperbolic metric of curvature -1 on $\mathbb{H}^{2}$ ) in terms of the signature of $\Gamma$. A second application of that formula shows that $\Gamma_{n}$ has signature $(n, 0)$ as required.

Now $\Gamma_{n}$ is a normal subgroup of $\Gamma$, so conjugation by $C$ determines an automorphism of the closed Riemann surface $\mathbb{H}^{2} / \Gamma_{n}$, which in turn determines an automorphism of $\mathbf{T}\left(\Gamma_{n}\right)$. The fixed point set of that automorphism is precisely $\mathbf{T}(\Gamma)$, and the inclusion map from $\mathbf{T}(\Gamma)$ to $\mathbf{T}\left(\Gamma_{n}\right)$ is isometric. (See $\S 2$ of [9] and Lemma 5.1 of Kravetz [22].)

Suppose that $h: \mathbf{T}(\Gamma) \rightarrow \mathscr{M}\left(\Gamma_{n}\right)$ is a holomorphic lifting of the inclusion map of $\mathbf{T}(\Gamma)$ in $\mathbf{T}\left(\Gamma_{n}\right)$. Thus $\Phi \circ h$ is the identity map on $\mathbf{T}(\Gamma)$. We may assume that $h(0)=0$. We must show that such an $h$ cannot exist. Since $\Phi: \mathscr{M}(\Gamma) \rightarrow \mathbf{T}(\Gamma)$ has no holomorphic sections (see [7]), it suffices to show that $h(\varphi) \in \mathscr{M}(\Gamma)$ for all $\varphi \in \mathbf{T}(\Gamma)$.

Choose any $\varphi \neq 0$ in $\mathbf{T}(\Gamma)$, and choose a holomorphic isometry $f: \Delta \rightarrow$ $\mathbf{T}(\Gamma)$ with $f(0)=0$ and $f\left(t_{0}\right)=\varphi$ for some point $t_{0}$ in the interval $(0,1)$. We can interpret $f$ as a holomorphic isometry into $\mathbf{T}\left(\Gamma_{n}\right)$, and conclude that $h \circ f: \Delta \rightarrow \mathscr{M}\left(\Gamma_{n}\right)$ is the unique holomorphic lift of $f$ that maps 0 to 0 . But we know that there is a holomorphic map $g: \Delta \rightarrow \mathscr{M}(\Gamma)$ such that $g(0)=0$ and $\Phi \circ g=f$. Since $\mathscr{M}(\Gamma) \subset \mathscr{M}\left(\Gamma_{n}\right)$, we must have $h \circ f=g$. In particular $h(\varphi)=g\left(t_{0}\right) \in \mathscr{M}(\Gamma)$ as required.

\section{APPENDIX}

Slodkowski [33] considers only holomorphic motions such that the set $E$ and the values of the motion $f$ are contained in the finite plane $\mathbb{C}$. It is easy to reduce the general case to the case of the finite plane, but a little care is necessary since two meromorphic functions can have intersecting graphs even if their difference has no zeros. To achieve the reduction, choose any point $z_{0}$ in $E$ and let $f_{0}: \Delta \rightarrow \widehat{\mathbb{C}}$ be the holomorphic mapping $f\left(\cdot, z_{0}\right)$. Then either $f_{0}$ is a meromorphic function on $\Delta$ or $f_{0} \equiv \infty$. In either case we can write $f_{0}=\varphi / \psi$, where $\varphi$ and $\psi$ are ( $\mathbb{C}$-valued) holomorphic functions on $\Delta$ with no common zeros.

Choose holomorphic functions $\alpha$ and $\beta$ on $\Delta$ such that $\beta \psi-\alpha \varphi=1$ identically on $\Delta$ (see Theorem 15.15 of [32] or Lemma 3.5.6 of [2]). For $t$ in $\Delta$, let $A_{t}$ be the Möbius transformation

$$
A_{t}(w)=\frac{\alpha(t) w-\beta(t)}{\psi(t) w-\varphi(t)}, \quad w \in \widehat{\mathbb{C}},
$$

and observe that $A_{t}\left(f\left(t, z_{0}\right)\right)=\infty$ for all $t$ in $\Delta$. Next, define a holomorphic motion $g$ of the set $A_{0}(E)$ by putting

$$
g\left(t, A_{0}(z)\right)=A_{t}(f(t, z)) \text { for all }(t, z) \in \Delta \times E,
$$

so that $g(t, \infty)=\infty$ for all $t$ in $\Delta$. Since $g$ takes only finite values on the set $\Delta \times\left(A_{0}(E)-\{\infty\}\right)$, Slodkowski's theorem permits us to extend the motion 
$g$ to a motion of $\widehat{\mathbb{C}}$. The desired extension of $f$ is then given by

$$
f(t, z)=A_{t}^{-1}\left(g\left(t, A_{0}(z)\right)\right) \text {. }
$$

\section{REFERENCES}

1. K. Astala and G.J. Martin, Holomorphic motions, (to appear).

2. C.A. Berenstein and R. Gay, Complex variables, an introduction, Graduate Texts in Math., vol. 125, Springer-Verlag, 1991.

3. L. Bers, Extremal quasiconformal mappings, Advances in the Theory of Riemann Surfaces, Ann. of Math. Stud., no. 66, Princeton Univ. Press, Princeton, NJ, 1971, pp. 27-52.

4. _ Holomorphic families of isomorphisms of Möbius groups, J. Math. Kyoto Univ. 26 (1986), 73-76.

5. L. Bers and H.L. Royden, Holomorphic families of injections, Acta Math. 157 (1986), 243257.

6. S. Dineen, The Schwarz lemma, Oxford Univ. Press, Oxford, 1989.

7. C.J. Earle, On holomorphic cross-sections in Teichmüller spaces, Duke Math. J. 36 (1969), 409-416.

8. (1970), 109-113.

9. $\ldots$ On the moduli of closed Riemann surfaces with symmetries, Advances in the Theory of Riemann Surfaces, Ann. of Math. Stud., no. 66, Princeton Univ. Press, Princeton, NJ, 1971, pp. 119-130.

10. Teichmüller theory, Discrete Groups and Automorphic Functions, Academic Press, 1977, pp. 143-162.

11. C.J. Earle and J. Eells, Jr., On the differential geometry of Teichmüller spaces, J. Analyse Math. 19 (1967), 35-52.

12. C.J. Earle and I. Kra, On sections of some holomorphic families of closed Riemann surfaces, Acta Math. 137 (1976), 49-79.

13. H.M. Farkas and I. Kra, Riemann surfaces, 2nd ed., Graduate Texts in Math., vol. 71, Springer-Verlag, New York, Heidelberg and Berlin, 1992.

14. F.P. Gardiner, Teichmüller theory and quadratic differentials, Wiley-Interscience, New York, 1987.

15. R.S. Hamilton, Extremal quasiconformal mappings with prescribed boundary values, Trans. Amer. Math. Soc. 138 (1969), 399-406.

16. L.A. Harris, Schwarz-Pick systems of pseudometrics for domains in normed linear spaces, Advances in Holomorphy, North-Holland Math. Studies, vol. 34, North-Holland, Amsterdam, 1979, pp. 345-406.

17. M. Hervé, Analyticity in infinite dimensional spaces, de Gruyter, Berlin, 1989.

18. E. Hille and R. Phillips, Functional analysis and semi-groups, Amer. Math. Soc. Colloq. Publ., vol. 32, Amer. Math. Soc., Providence, RI, 1957.

19. I. Kra, On new kinds of Teichmüller spaces, Israel J. Math 6 (1973), 237-25.

20. _ On the Nielsen-Thurston-Bers type of some of some self-maps of Riemann surfaces, Acta Math. 146 (1981), 231-270.

21. $\ldots$, Families of univalent functions and Kleinian groups, Israel J. Math. 60 (1987), 89127.

22. S. Kravetz, On the geometry of Teichmüller spaces and the structure of their modular groups, Ann. Acad. Sci. Fenn. Ser. A I Math. 278 (1959), 1-35.

23. S.L. Krushkal', Extremal problems for conformal and quasiconformal mappings of Riemann surfaces, Sibirsk Mat. Z. 14 (1973), 582-598 and 694.

24. R. Mañé, P. Sad, and D. Sullivan, On dynamics of rational maps, Ann. Sci. École Norm. Sup. 16 (1983), 193-217. 
25. C. McMullen, Amenability, Poincaré series and quasiconformal maps, Invent. Math. 97 (1989), 95-127.

26. S. Nag, Non-geodesic discs embedded in Teichmüller spaces, Amer. J. Math. 104 (1982), 399-408.

27. 1988.

28. B. O'Byrne, On Finsler geometry and applications to Teichmüller spaces, Advances in the Theory of Riemann Surfaces, Ann. of Math. Stud., no. 66, Princeton Univ. Press, Princeton, NJ, 1971, pp. 317-328.

29. E. Reich and K. Strebel, Extremal quasiconformal mappings with given boundary values, Contributions to Analysis, Academic Press, San Diego, 1974, pp. 375-391.

30. H.L. Royden, Automorphisms and isometries of Teichmüller space, Advances in the theory of Riemann Surfaces, Ann. of Math. Stud., no. 66, Princeton, Univ. Press, Princeton, NJ, 1971, pp. 369-383.

31. 270.

32. W. Rudin, Real and complex analysis, 3rd ed., McGraw-Hill, New York, 1987.

33. Z. Slodkowski, Holomorphic motions and polynomial hulls, Proc. Amer. Math. Soc. 111 (1991), 347-355.

34. Invariant extensions of holomorphic motions, Abstracts Amer. Math. Soc. 13 (1992), 259.

35. K. Strebel, On quasiconformal mappings of open Riemann surfaces, Comment. Math. Helv. 53 (1978), 301-321.

36. D. Sullivan, Quasiconformal homeomorphisms and dynamics. I: Solution of the Fatou-Julia problem on wandering domains, Ann. of Math. (2) 122 (1985), 401-418.

37. _ـ Quasiconformal homeomorphisms and dynamics. II: Structural stability implies hyperbolicity for Kleinian groups, Acta Math. 155 (1985), 243-260.

38. _ Quasiconformal homeomorphisms and dynamics. III: Topological conjugacy classes of analytic diffeomorphisms (to appear).

39. D. Sullivan and W.P. Thurston, Extending holomorphic motions, Acta Math. 157 (1986), 243-257.

40. H. Tanigawa, Holomorphic families of geodesic discs in infinite dimensional Teichmüller spaces, Nagoya Math. J. 127 (1992), 117-128.

41. Li Zhong, Nonuniqueness of geodesics in infinite dimensional Teichmüller spaces, Complex Variables Theory Appl. 16 (1991), 261-272.

42. A. Douady, Prolongement de mouvements holomorphes [d'après Slodkowski et autres], Séminaire N. Bourbaki, 1993-1994, Exposé 775, pp. 1-12.

Department of Mathematics, Cornell University, Ithaca, New York 14853

Department of Mathematics, State University of New York at Stony Brook, Stony BROOK, NEW YORK 11794

Siberian Division of the USSR ACADEmy of Sciences, Novosibirsk, USSR

Current address, S. L. Krushkal': Department of Mathematics and Computer Science, Bar-Ilan University, 52900 Ramat-Gan, Israel 